In der Rubrik „Literatur kompakt" werden die wichtigsten Originalarbeiten aus der internationalen Fachliteratur referiert.

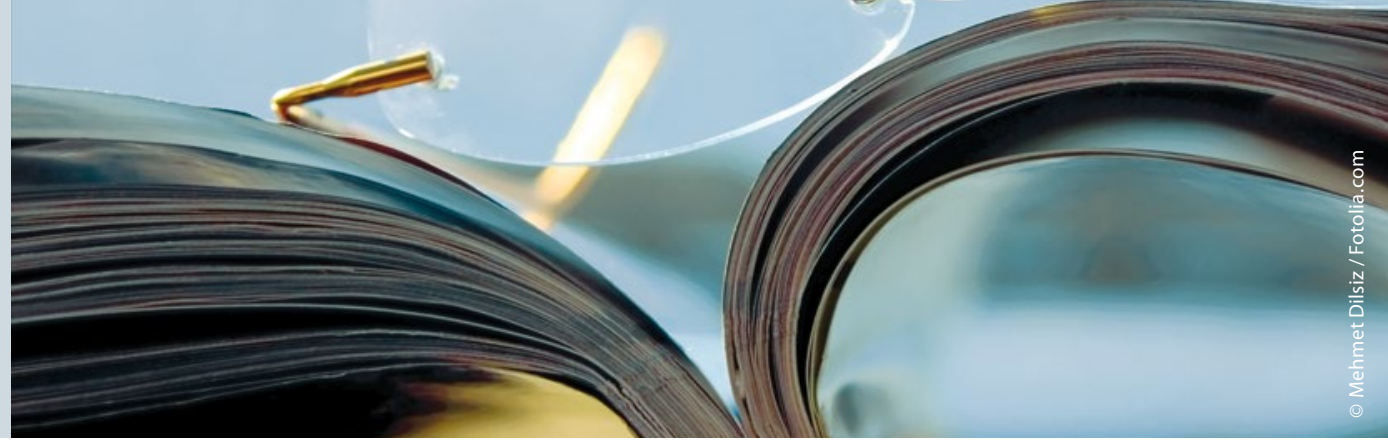

\section{Harnröhrenrekonstruktion: Erfolgreiche Rezidivtherapie}

\author{
Wenn eine Harnröhrenrekonstruktion aufgrund einer Striktur mit Gewebe der \\ Mundschleimhaut wiederholt werden muss, gleicht das Therapieergebnis \\ wahrscheinlich jenem nach der ersten Behandlung.
}

$\mathrm{D}$ ie Erfolgsrate nach primärer Urethroplastik liegt mit 79 bis $95 \%$ - bei einer beobachteten Langzeitrate $(>10$ Jahre) von immer noch $70 \%$ - sehr hoch, wie Urologen um Dr. Clemens M. Rosenbaum vom Universitätsklinikum Hamburg-Eppendorf und seine Kollegen berichten.

Nach wiederholter Urethroplastik liege die strikturfreie Überlebensrate zwischen 67 und $92 \%$ bei einem medianen Follow-up von 55 Monaten. Diskussionen gebe es allerdings über den Stellenwert der Morbidität nach einer erneuten Urethroplastik unter Verwendung von Gewebe der Mundschleimhaut.

Bisher gab es keine veröffentlichten Daten zum Therapieerfolg nach einer wiederholten Verpflanzung von Mundschleimhaut. Um ihre Hypothese zu überprüfen, dass die erneute Urethroplastik gleichermaßen erfolgreich ist, werteten die Hamburger Urologen um Rosenbaum nun retrospektiv die Befunde der Patienten an ihrem Zentrum der Maximalversorgung aus. Dort nehmen sie jährlich etwa 200 Urethroplastiken vor.

Von den 825 Eingriffen zwischen 2009 und 2014 handelte es sich bei 55 Patienten um eine erneute Urethroplastik nach einem Strikturrezidiv. Die Befunde von 50 Patienten ließen sich schließlich von Rosenbaum et al. für die Studie verwerten. Bei allen Patienten war die bukkale Schleimhaut auf der kontralateral zum ersten Eingriff liegenden Wangenseite entnommen worden. Die verwendeten, meist ovoidförmigen Gewebeareale konnten bis zur Unterlippe reichen.

Bei jedem zweiten Patienten (55 \%) mit einem Rezidiv war der primäre Eingriff an einer anderen Klinik vorgenommen worden. Ein erneuter Eingriff war bei den meisten Patienten $(71,4 \%)$ wegen einer bulbären Striktur erforderlich. Seltener war eine penile Striktur für den

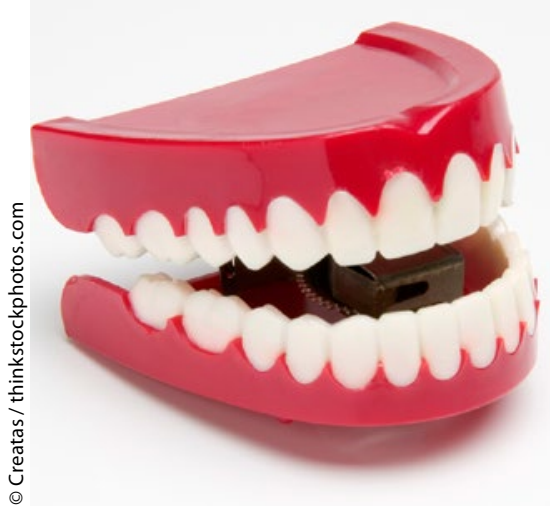

Der Therapieerfolg einer erneuten Urethroplastik unter Verwendung von Mundschleimhautgewebe scheint einer neuen Studie zufolge zufriedenstellend. neuerlichen Eingriff ursächlich. Bei $18 \%$ war es im Mittel nach 13,8 Monaten zum Rezidiv gekommen. Nach einem Jahr sowie nach zwei und drei Jahren lag der Anteil der Patienten ohne eine erneute Harnröhrenverengung bei $91,2 \%, 86,2 \%$ beziehungsweise $80,8 \%$. Nur $3 \%$ der Patienten klagten über veränderten Speichelfluss oder über Schwierigkeiten beim Öffnen des Mundes. 13,5 \% der Patienten gaben an, ein ausgeprägtes bis sehr starkes Taubheitsgefühl im Mund zu haben.

Drei von vier Patienten meinten, ihre Lebensqualität habe sich durch den erneuten Eingriff verbessert. 13,6 \% der Patienten fühlten sich nach wiederholter Urethroplastik im täglichen Leben mäßig, immerhin noch $2,7 \%$ sehr stark beeinträchtigt.

Fazit: Insgesamt lag die Erfolgsrate des erneuten Eingriffs, definiert als Anteil der Patienten ohne Harnröhrenverengung, bei $82 \%$ bei einem durchschnittlichen Follow-up von 25,6 Monaten, was zufriedenstellend sei, so die Urologen um Rosenbaum. Auch der Anteil der Patienten mit sexueller Dysfunktion war mit $14,3 \%$ ähnlich niedrig wie nach der Primärtherapie mit etwa $12 \%$. Weder die Primärtherapie noch Lokalisation und Länge der Striktur entpuppten sich als Risikofaktoren für den erneuten Eingriff. Einschränkend weisen die Ärzte darauf hin, dass es sich nur um eine kleine Patientenzahl handelt und das Follow-up relativ kurz ist.

Peter Leiner

Rosenbaum CM et al. Redo-buccal mucosa graft urethroplasty: success rate, oral morbidity and functional outcome. BJU Int. 2016;doi: 10.1111/ bju.13528. 